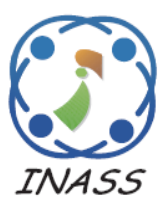

\title{
Software Cost Estimation by Optimizing COCOMO Model Using Hybrid BATGSA Algorithm
}

\author{
Deepak Nandal ${ }^{1 *}$ \\ Om Prakash Sangwan ${ }^{1}$ \\ ${ }^{1}$ Department of Computer Science and Engineering, \\ Guru Jambheshwar University of Science and Technology, Hisar, Haryana, India \\ * Corresponding author’s Email: dr.deepaknandalgju@ gmail.com
}

\begin{abstract}
This paper estimates the effort for software by optimizing the COnstructive COst MOdel (COCOMO) model parameters using hybrid BATGSA (Bat inspired Gravitational Search Algorithm) algorithm. The performance of the COCOMO model completely depends upon its parameters which can be optimized by using meta-heuristic algorithms. This paper uses hybrid BATGSA algorithm which hybrids the improved bat algorithm with the gravitation search algorithm (GSA) to optimize the COCOMO model. The bat algorithm demonstrates the hunting and routing behavior of the bat which is improved by using a random walk in the exploration phase. The exploration phase is further improved by using GSA as gravitation force affects the velocity of the bat. The algorithm has been analyzed on four NASA datasets downloaded from promise repository. The comparison of the algorithm has been made with existing three states of art techniques i.e. COCOMO model, BAT algorithm, Improved BAT(IBAT) algorithm by using normalized error as a parameter. The reduction in error ranges from $2 \%$ to $10 \%$ on different dataset as compared other state of art algorithms proves the significance of proposed algorithm.
\end{abstract}

Keywords: NASA project dataset, COCOMO, BAT, GSA, Optimization.

\section{Introduction}

Software cost estimation is a critical process as it is required to estimate the cost of the project at the initial stage of the project. The cost estimation is required to compute the budget and the resources required for the project $[1,2]$. The cost of the project depends upon the efforts done which include the number of reviews, efficiency during implementation and pre-development processing, etc [3]. The Constructive Cost Model (COCOMO) is a model to determine the cost of the software. This model uses a basic regression formula for estimating cost, effort, and schedule for software projects based on features of the project. The model calculates the efforts as $E=a \times(K L O C) b$, here KLOC shows the kilo line of code and $a, b$ are the constants which depends upon the type of software [4]. This model can be optimized by using meta-heuristic algorithm [5]. Different authors have applied the various metaheuristic algorithm to improve the performance of the COCOMO model. The author of [6] has applied a genetic algorithm to optimize the parameters of COCOMO model while the author of [1] has applied differential evolution algorithm for the same. The author of [7] has applied BAT algorithm to improve the performance of cost estimation by COCOMO. The author of [7] shows the reduction in error by optimizing the COCOMO model using BAT algorithm. The BAT algorithm shows the better result as compared other existing state of the art techniques but BAT algorithm search locally in the exploration phase which opens scope for the improvement. This paper optimizes the estimation using COCOMO model by using hybrid BATGSA algorithm. The hybrid BATGSA algorithm has better convergence towards the global optima as it has improved exploration phase as compared to BAT algorithm, resulting in better optimization of COCOMO model. This paper further has been classified in five sections. The next section i.e. section 2 describes the improved BAT algorithm 
which is followed by the description of Gravitation search algorithm (GSA) in section 3. The Hybrid BATGSA algorithm has been described in section 4 while the results and corresponding discussion is done in section 5 .

\section{Improved BAT algorithm for cost estimation}

Bat algorithm performs well for the various applications [8, 9]. This algorithm automatically switches from the exploration phase i.e. global search, to the exploitation phase i.e. local search. The main limitation of the algorithm is that it mainly searches locally even in the exploration phase $[7,9]$. In the exploration phase change in the frequency is the parameter for the global search which restricts the search locally mainly. This behavior has been changed by introducing the random move nature to the bat resultant velocity updation is given by Eq. (1). Suppose there are $n$ bats with position vector $\mathrm{P}[1: \mathrm{n}]$ and velocity vector $\mathrm{V}[1: \mathrm{n}]$ i.e. $P_{a}^{t}, V_{a}^{t}$ denotes the position and velocity of the $\mathrm{a}^{\text {th }}$ bat at $\mathrm{t}^{\text {th }}$ time. Moreover, there is a frequency vector $f[1: n]$ that denotes the frequency of each bat which lies between $f_{\min }$ and $f_{\max }$ The velocity of each bat is adjusted according to frequency using the equation

$$
V_{a}^{t}=\left\{\begin{array}{c|c}
V_{a}^{t-1}+\left(P_{a}^{t-1}-P^{b}\right) f_{a} & p r<0.5 \\
V_{a}^{t-1}+\left(P_{a}^{t-1}-P^{r}\right) \mathrm{Xpr} & \text { else }
\end{array}\right.
$$

Here $\mathrm{pr}$ is the probability which is generated randomly by using a random function and $P^{r}$ is position at any random time stamp. This updation strength the exploration phase and improves the global search. Correspondingly position update can be given by Eq. (2) to improve the impact of velocity on the position.

$$
P_{a}^{t}=\left\{\begin{array}{c|c}
P_{a}^{t-1}+V_{a}^{t} & p r<0.5 \\
P_{a}^{t-1}+V_{a}^{t} f_{a} & \text { else }
\end{array}\right.
$$

The Eq. (2) updates the position of the bat according to the velocity update. The local search phase of the algorithm is powerful enough to exploit the search space. The improved bat algorithm is as follow:

IBAT Algorithm (P, V, f, F, emr $\left.{ }^{0}, \mathrm{~L}\right)$ :

Here, $\mathrm{P}, \mathrm{V}, \mathrm{f}$ are the position, velocity and frequency vector for $\mathrm{n}$ bats respectively. The $\mathrm{F}$ is the fitness function to evaluate the fitness of the solution generated by the corresponding position. The $\mathrm{L}$ is the loudness vector and $\mathrm{emr}^{0}$ is initial emission rate.
1. Initiate iteration $=1$

2. $\mathrm{P}^{\mathrm{b}}=\mathrm{P}_{1}$

3. For $\mathrm{i}=2: \mathrm{n}$
a. If $\mathrm{F}\left(\mathrm{P}_{\mathrm{i}}\right)>\mathrm{F}\left(\mathrm{P}^{\mathrm{b}}\right)$
b. $\mathrm{P}^{\mathrm{b}}=\mathrm{P}_{\mathrm{i}}$
c. End if

End

4. While iteration $<$ max_iteration

$$
\begin{aligned}
& \text { a. } f_{\text {iteration }}=f_{\text {min }}+\left(f_{\max }-f_{\text {min }}\right) \alpha \\
& \text { b. } \quad \text { pr=rand } \\
& \text { c. } \quad r=\operatorname{rand}(1, n)
\end{aligned}
$$

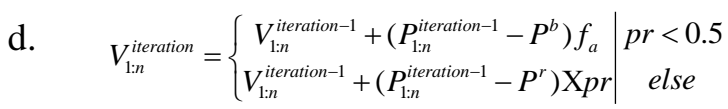

$$
\begin{aligned}
& \text { e. } \quad P_{1: n}^{\text {iteration }}=\left\{\begin{array}{c}
P_{1: n}^{\text {iteration-1 }}+V_{1: n}^{\text {iteration }} \\
P_{1: n}^{\text {iteration-1 }}+V_{1: n}^{\text {iteration }} f_{1: n}
\end{array} \mid \begin{array}{c}
p r<0.5 \\
\text { else }
\end{array}\right. \\
& P_{1: n}^{\text {iteration }}=P_{1: n}^{\text {iteration }-1}+\varepsilon L^{\text {iteration }}
\end{aligned}
$$

End if

$$
\begin{gathered}
\text { g. } \quad \text { If } \mathrm{F}\left(P_{1: n}^{\text {iteration }}\right)>\mathrm{F}\left(P_{1: n}^{\text {iteration-1 }}\right) \\
P_{1: n}^{\text {iteration }}=P_{1: n}^{\text {iteration }-1} \\
\text { Else } \\
L_{1: n}^{\text {iteration }}=L_{1: n}^{\text {iteration-1 }} \beta \\
\quad e m r_{1: n}^{\text {iteration }}=e m r_{1: n}^{0}\left(1-e^{-\lambda(\text { iteration-1) })}\right)
\end{gathered}
$$

End if

h. For $\mathrm{i}=1: \mathrm{n}$

$$
\begin{aligned}
\text { i. } & \text { If } F\left(P_{i}\right)>F\left(P^{b}\right) \\
\text { ii. } & P^{b}=P_{i} \\
\text { iii. } & \text { End if }
\end{aligned}
$$

End

i. iteration++
End while

The above algorithm gives the process of improved bat algorithm [10,11]. This algorithm has been applied to the cost estimation of software by using the objective function given by Eq. (3) 


$$
F(P)=\left(\sum_{i=1}^{n}\left(\text { actual }_{i}-\text { predicted }_{i}\right) / \text { actual }_{i}\right) / n
$$

which calculates the mean relative error which depends upon the actual and the predicted estimation. The performance of the algorithm can be improved discussed in next section.

\section{Gravitational search algorithm}

The gravitational search algorithm uses the law of gravity given by Newton [12]. According to Newton every particle attracts another particle with a gravitational force that can be given by Eq. (4).

$$
F=G \frac{M_{1} M_{2}}{D^{2}}
$$

Here, $G$ is the gravitational constant, $D$ is the distance between two objects having masses $M_{1}$ and $\mathrm{M}_{2}$ respectively. The details of GSA are as follow. Suppose we have $\mathrm{n}$ objects in dimensions then position matrix for objects can be given by Eq. (5).

$$
O=\left[\begin{array}{cccc}
O_{1}^{1} & O_{1}^{2} & L & O_{1}^{d} \\
O_{2}^{1} & O_{2}^{2} & L & O_{2}^{d} \\
M & M & M & M \\
O_{n}^{1} & O_{n}^{2} & L & O_{n}^{d}
\end{array}\right]
$$

Here, $O_{i}^{j}$ represents the position of $\mathrm{i}^{\text {th }}$ object in the $\mathrm{j}^{\text {th }}$ dimension. At a particular time stamp say $\mathrm{t}$ the force acting on object $\mathrm{p}$ from object $\mathrm{q}$ can be given by Eq. (6).

$$
F_{p q}^{1: d}(t)=G(t) \frac{M_{p}^{a}(t) M_{q}^{p}(t)}{D_{p q}(t)+\varepsilon}\left(O_{i}^{i: d}(t)-O_{j}^{1: d}(t)\right)
$$

Here $M_{p}^{a}(t), M_{q}^{p}(t)$ are active and passive gravitational masses of object $\mathrm{p}$ and q respectively. $\varepsilon$ is a small constant with value $2^{-52}, \quad D_{p q}(t)$ is the distance between object $\mathrm{p}$ and object $\mathrm{q}$ which is given by Eq. (7).

$$
D_{p q}(t)=\sqrt{\sum_{i=1}^{d}\left(O_{p}^{i}-O_{q}^{i}\right)^{2}}
$$

Overall the total force acting on any object $\mathrm{p}$ can be given by Eq. (8)

$$
F_{p}^{1: d}(t)=\sum_{i=1, i \neq p}^{n} F_{p i}^{1: d} \mathrm{Xrand}_{i}
$$

So the acceleration is given by Eq. (9).

$$
a_{p}^{1: d}(t)=\frac{F_{p}^{1: d}(t)}{O_{p}(t)}
$$

Here, $O_{p}(t)$ is the inertia mass of object p.

This acceleration is used to update the velocity of the object which affects the position of an object demonstrated by Eqs. (10) and (11).

$$
v_{p}^{1: d}(t+1)=v_{p}^{1: d}(t) \mathrm{Xrand}+a_{p}^{1: d}(t)
$$

and

$$
O_{p}^{1: d}(t+1)=O_{p}^{1: d}(t)+v_{p}^{1: d}(t+1)
$$

The whole process is repeated for the updated position for the given number of iterations or until fulfilling the stopping criteria. The overall GSA algorithm can be given as follow.

\section{GSA (O, M, Fit)}

The algorithm uses $\mathrm{O}$ as the object position matrices with $\mathrm{M}$ as the mass metric containing mass of each object. Fit is the fitness function.

1. Initiate iteration $=1$

2. $O=\left[\begin{array}{cccc}O_{1}^{1} & O_{1}^{2} & L & O_{1}^{d} \\ O_{2}^{1} & O_{2}^{2} & L & O_{2}^{d} \\ M & M & M & M \\ O_{n}^{1} & O_{n}^{2} & L & O_{n}^{d}\end{array}\right]$

3. While iteration $<$ max_iteration

a. $\operatorname{Fit}(t)=$ $\left[\begin{array}{cccc}\text { Fit }\left(O_{1}^{1}(t)\right) & \text { Fit }\left(O_{1}^{2}(t)\right) & L & \text { Fit }\left(O_{1}^{d}(t)\right) \\ \text { Fit }\left(O_{2}^{1}(t)\right) & \text { Fit }\left(O_{2}^{2}(t)\right) & L & \text { Fit }\left(O_{2}^{d}(t)\right) \\ M & M & M & M \\ \text { Fit }\left(O_{n}^{1}(t)\right) & \text { Fit }\left(O_{n}^{2}(t)\right) & L & \text { Fit }\left(O_{n}^{d}(t)\right)\end{array}\right]$

b. $\quad D_{p q}(t)=\sqrt{\sum_{i=1}^{d}\left(O_{p}^{i}-O_{q}^{i}\right)^{2}}$

c. $\quad F_{p q}^{\mathrm{ld} d}(t)=G(t) \frac{M_{p}^{a}(t) M_{q}^{p}(t)}{D_{p q}(t)+\varepsilon}\left(O_{i}^{i, d}(t)-O_{j}^{1: d}(t)\right)$

d. $\quad F_{p}^{1: d}(t)=\sum_{i=1, i \neq p}^{n} F_{p i}^{1: d} \mathrm{Xrand}_{i}$ 
e. $\quad a_{p}^{1: d}(t)=\frac{F_{p}^{1: d}(t)}{O_{p}(t)}$

f. $\quad v_{p}^{1: d}(t+1)=v_{p}^{1: d}(t) \mathrm{Xrand}+a_{p}^{1: d}(t)$

g. $\quad O_{p}^{1: d}(t+1)=O_{p}^{1: d}(t)+v_{p}^{1: d}(t+1)$

h. If $\operatorname{Fit}(\mathrm{t})<\operatorname{Fit}(\mathrm{t}+1)$

$$
O_{p}^{1: d}(t+1)=O_{p}^{1: d}(t)
$$

end

End while

\section{Return best $(\mathrm{F})$}

The above algorithm performs the optimization. The fitness has been given by Eq. (3) to determine the software cost estimation. The improved bat algorithm is hybridized by using gravitational search algorithm discussed in next section.

\section{Hybrid BATGSA algorithm}

In the real-time the gravitational force as the impact the velocity of the BAT. in this work to improve the exploration phase of the algorithm the velocity factor has been affected by the gravitational force also. This improves the global search capability of the algorithm resulting better convergence for optimization. The concept can be easily understood by following algorithm:

BATGSA Algorithm (P, V, M, f, Fit, emr ${ }^{0}$, L)

Here, $\mathrm{P}, \mathrm{V}, \mathrm{M}, \mathrm{f}$ are the position, velocity, mass and frequency vector for $\mathrm{n}$ bats respectively. The Fit is the fitness function to evaluate the fitness of the solution generated by corresponding position. The $\mathrm{L}$ is the loudness vector and $\mathrm{emr}^{0}$ is initial emission rate.

1. Initiate iteration $=1$

2. $P=\left[\begin{array}{cccc}P_{1}^{1} & P_{1}^{2} & L & P_{1}^{d} \\ P_{2}^{1} & P_{2}^{2} & L & P_{2}^{3} \\ M & M & M & M \\ P_{n}^{1} & P_{n}^{2} & L & P_{n}^{d}\end{array}\right]$

3. Fit(iteration $)=\left[\begin{array}{cccc}F i t\left(P_{1}^{1}(\text { iteration })\right) & \text { Fit }\left(P_{1}^{2}(\text { iteration })\right) & L & \left.F i t\left(P_{1}^{d} \text { (iteration }\right)\right) \\ F i t\left(P_{2}^{1}(\text { iteration })\right) & F i t\left(P_{2}^{2}(\text { iteration })\right) & L & F i t\left(P_{2}^{d}(\text { iteration })\right) \\ M & M & M & M \\ F i t\left(P_{n}^{1}(\text { iteration })\right) & F i t\left(P_{n}^{2}(\text { iteration })\right) & L & F i t\left(P_{n}^{d}(\text { iteration })\right)\end{array}\right]$

4. $\mathrm{P}^{\mathrm{b}}=\mathrm{P}_{1}$

5. For $\mathrm{i}=2: \mathrm{n}$
a. If $\operatorname{Fit}\left(\mathrm{P}_{\mathrm{i}}\right)>\operatorname{Fit}\left(\mathrm{P}^{\mathrm{b}}\right)$
b. $\mathrm{P}^{\mathrm{b}}=\mathrm{P}_{\mathrm{i}}$
c. End if

End

6. While iteration<max_iteration
a. $f_{\text {iteration }}=f_{\text {min }}+\left(f_{\text {max }}-f_{\text {min }}\right) \alpha$
b. $\mathrm{pr}=$ rand
c. $r=\operatorname{rand}(1, \mathrm{n})$ 
d. $\quad V_{1: n}^{\text {iteration }}=\left\{\begin{array}{c|c}V_{1: n}^{\text {iteration }-1}+\left(P_{1: n}^{\text {iteration- }-1}-P^{b}\right) f_{a} & p r<0.5 \\ V_{1: n}^{\text {iteration-1 }}+\left(P_{1: n}^{\text {iteration-1 }}-P^{r}\right) \mathrm{Xpr} & \text { else }\end{array}\right.$

e. $\quad P_{1: n}^{\text {iteration }}=\left\{\begin{array}{c}P_{1: n}^{\text {iteration-1 }}+V_{1: n}^{\text {iteration }} \\ P_{1: n}^{\text {iteration-1 }}+V_{1: n}^{\text {iteration }} f_{1: n}\end{array} \mid \begin{array}{c}\text { else }\end{array}\right.$

f. If rand $>$ emriteration- 1

End if

$$
P_{1: n}^{\text {iteration }}=P_{1: n}^{\text {iteration-1 }}+\varepsilon L^{\text {iteration }}
$$

g. For $\mathrm{p}=1: \mathrm{n}$

i. For $\mathrm{q}=1: \mathrm{n}$

$$
\begin{aligned}
& D_{p q}(\text { iteration }-1)=\sqrt{\sum_{i=1}^{d}\left(P_{p}^{i}-P_{q}^{i}\right)^{2}} \\
& F_{p q}^{1: d}(\text { iteration }-1)=G(\text { iteration }) \frac{M_{p}^{a}(t) M_{q}^{p}(t)}{D_{p q}(t)+\varepsilon}\left(P_{p}^{i: d}(\text { iteration })-P_{q}^{1: d}(t)\right)
\end{aligned}
$$

End

ii. $F_{p}^{1: d}($ iteration -1$)=\sum_{i=1, i \neq p}^{n} F_{p i}^{1: d} \mathrm{Xrand}_{i}$

iii. $\quad a_{p}^{1: d}($ iteration -1$)=\frac{F_{p}^{1: d}(\text { iteration })}{P_{p}(\text { iteration })}$

iv. $\quad v_{p}^{1: d}($ iteration $)=v_{p}^{1: d}($ iteration -1$) \mathrm{Xrand}+a_{p}^{1: d}($ iteration -1$)$

v. $\quad P_{p}^{1: d}($ iteration $)=P_{p}^{1: d}($ iteration -1$)+v_{p}^{1: d}($ iteration $)$

End

h. $\quad$ If $\operatorname{Fit}\left(P_{1: n}^{\text {iteration }}\right)>\operatorname{Fit}\left(P_{1: n}^{\text {iteration-1 }}\right)$

$$
P_{1: n}^{\text {iteration }}=P_{1: n}^{\text {iteration- } 1}
$$

Else

$$
\begin{aligned}
& L_{1: n}^{\text {iteration }}=L_{1: n}^{\text {iteration-1 }} \beta \\
& e m r_{1: n}^{\text {iteration }}=e m r_{1: n}^{0}\left(1-e^{-\lambda(\text { iteration-1) }}\right)
\end{aligned}
$$

End if

i. For $\mathrm{i}=1: \mathrm{n}$
i. If $\mathrm{F}\left(\mathrm{P}_{\mathrm{i}}\right)>\mathrm{F}\left(\mathrm{P}^{\mathrm{b}}\right)$
ii. $\mathrm{P}^{\mathrm{b}}=\mathrm{P}_{\mathrm{i}}$
iii. End if

End

j. iteration++

End while 
The above algorithm updates the position of the bat firstly on the basis of frequency of the sound then this position is updated on the basis of the gravitational force on bat for the global search while the local search still depends upon the loudness of the sound. This algorithm produces exploration as well as exploitation search so the optimization must converge towards the global optima. The objective function given in Eq. (3) has been used for the cost estimation. The implementation of algorithm and the result analysis has been discussed in next section.

\section{Results and discussion}

The algorithm discussed in previous section has been implemented using MATLAB. The analysis has been done on 4 datasets, 2 are the NASA datasets containing 60 and 93 projects respectively and other two are COCOMO81 and kemerer. All datasets has been downloaded from the promise data repository. The actual cost of each software project is available in the dataset which is compared with the estimated cost to calculate the mean relative error for each project. The comparison of the hybrid BATGSA algorithm is done with the COCOMO based cost estimation i.e. cost estimation without optimization [3] with the BAT [7] and improved BAT based optimization [11]. The remaining section of the paper uses Dataset1 for the cocomo81 dataset, Dataset2 for NASA dataset having 60 projects, Dataset3 for NASA dataset with 93 project and Dataset 4 for kemerer dataset. The software effort estimation for Dataset1 has been analyzed in Table1.

The effort estimation for each project in the Dataset 1 has been shown in the table 1 . The analysis Table 1 shows that the value of the effort converges towards the actual effort but for some project the estimation is producing higher error. This is because the algorithm reduces the mean absolute error in cost estimation by Eq. (8). The mean absolute error in the Dataset1 has been to 433.215 from 437.537(Improved BAT), 444.405 (Bat algorithm) which is reduced from 680.154 in COCOMO model. The normalized error in each project of Dataset1 has been analyzed in figure 1 . The normalized error has been calculated by Eq. (12).

$$
N E_{i}=a b s\left(\text { actual }_{i}-\text { predicted }_{i}\right) / \text { actual }_{i}
$$

Here, $\mathrm{NE}_{\mathrm{i}}$ is the normalized error for $\mathrm{i}$-th project which has been calculated by using actual and predicted effort of the project.
The Fig. 1 clearly shows that normalized error has been reduced by using hybrid BATGSA is more as compared to reduction by improving the bat algorithm and bat algorithm. This is due to the better optimization of COCOMO model by BATGSA algorithm which is due to better exploration phase of BATGSA algorithm.

The software cost/effort estimation for each undertaking in the Dataset 2 has been appeared in the table 2. The mean absolute error in the Dataset2 has been to 127.530 from 132.486 in improved BAT and 137.401 (Bat calculation) which is decreased from 400.985 in COCOMO display. The standardized error in each project of Dataset2 has been investigated in Fig. 2 which is computed by Eq. (12).

The Fig. 2 unmistakably connotes that normalized error has been reduced by hybrid BATGSA calculation is higher than the reduction done by improved bat and bat calculation due to avoidance of local minima by the BATGSA algorithm.

The effort estimation for each project in the Dataset3 has been shown in the Table 3. The analysis table 3 shows that the value of the effort converges towards the actual effort but for some project the estimation is producing higher error. This is because the algorithm reduces the mean absolute error in cost estimation by Eq. (3). The mean absolute error in the Dataset1 has been to 355.386 from 356.143(improved BAT), 365.164 (Bat algorithm) which is reduced from 618.412 in COCOMO model.

The Fig. 3 clearly shows that normalized error has been reduced by using hybrid BATGSA is more as compared to reduction by improving the bat algorithm and bat algorithm. This is due to the better optimization of COCOMO model by BATGSA algorithm which is due to better exploration phase of BATGSA algorithm.

The software cost/effort estimation for each undertaking in the Dataset4 has been appeared in the table 4. The mean absolute error in the Dataset4 has been to 2398.141 from 4564.934 in improved BAT and from 5528.158 (Bat calculation) which is decreased from 6804.450 in COCOMO display. The standardized error in each project of Dataset4 has been investigated in figure 4 which is computed by Eq. (12).

The Fig. 4 unmistakably connotes that normalized error has been reduced by hybrid BATGSA calculation is higher than the reduction done by improved bat and bat calculation. This is due to the velocity variation done to explore the search space appropriately. This shows that the 
hybrid BATGSA algorithm improves the estimation of the software effort.

Table 1. Analysis of effort estimation on Dataset 1

\begin{tabular}{|c|c|c|c|c|c|}
\hline $\begin{array}{l}\text { Project } \\
\text { Number }\end{array}$ & $\begin{array}{l}\text { Hybrid } \\
\text { BATGSA } \\
\text { Optimization }\end{array}$ & $\begin{array}{l}\text { IBAT } \\
\text { Optimization }\end{array}$ & $\begin{array}{l}\text { BAT } \\
\text { Optimization }\end{array}$ & СOCOMO & Actual \\
\hline 1 & 500.0036 & 454.2916 & 474.5296 & 3.134719 & 2040 \\
\hline 2 & 1446.034 & 1362.665 & 1443.515 & 3 & 1600 \\
\hline 3 & 594.5699 & 543.4377 & 568.9498 & 3 & 243 \\
\hline 4 & 246.9118 & 218.9643 & 226.593 & 3.134719 & 240 \\
\hline 5 & 56.58902 & 47.70643 & 48.41535 & 3 & 33 \\
\hline 6 & 12.0693 & 9.64865 & 9.593832 & 3 & 43 \\
\hline 7 & 22.16179 & 18.09085 & 18.13331 & 3 & 8 \\
\hline 8 & 80.70177 & 68.86921 & 70.22168 & 3.270061 & 1075 \\
\hline 9 & 114.0294 & 98.4732 & 100.8675 & 3 & 423 \\
\hline 10 & 109.801 & 94.69864 & 96.95262 & 3 & 321 \\
\hline 11 & 122.5342 & 106.0797 & 108.7624 & 3 & 218 \\
\hline 12 & 144.0569 & 125.4077 & 128.8548 & 3.270061 & 201 \\
\hline 13 & 93.0598 & 79.80509 & 81.52591 & 3 & 79 \\
\hline 14 & 8.758434 & 6.925093 & 6.856592 & 3.782814 & 60 \\
\hline 15 & 11.73347 & 9.371089 & 9.314369 & 3.782814 & 61 \\
\hline 16 & 19.31758 & 15.69484 & 15.70312 & 3 & 40 \\
\hline 17 & 10.73201 & 8.54502 & 8.483278 & 3 & 9 \\
\hline 18 & 1595.32 & 1508.428 & 1600.005 & 3.270061 & 11400 \\
\hline 19 & 6638.286 & 6591.914 & 7125.294 & 3 & 6600 \\
\hline 20 & 1479.077 & 1394.886 & 1478.089 & 3.270061 & 6400 \\
\hline 21 & 1222.389 & 1145.283 & 1210.54 & 3 & 2455 \\
\hline 22 & 524.7245 & 477.5438 & 499.1363 & 3.782814 & 724 \\
\hline 23 & 326.0589 & 291.9289 & 303.2134 & 3.782814 & 539 \\
\hline 24 & 387.982 & 349.452 & 363.7963 & 3 & 453 \\
\hline 25 & 148.4032 & 129.3233 & 132.9303 & 3.270061 & 523 \\
\hline 26 & 192.5427 & 169.2959 & 174.6185 & 3.270061 & 387 \\
\hline 27 & 31.28028 & 25.83853 & 26.01757 & 3.270061 & 88 \\
\hline 28 & 44.8975 & 37.55026 & 37.99172 & 3 & 98 \\
\hline 29 & 6.01046 & 4.69124 & 4.621749 & 3.782814 & 7.3 \\
\hline 30 & 5.51178 & 4.289229 & 4.220852 & 3.782814 & 5.9 \\
\hline 31 & 256.1027 & 227.4003 & 235.4368 & 3 & 1063 \\
\hline 32 & 1988.874 & 1894.85 & 2015.761 & 3 & 702 \\
\hline 33 & 165.9165 & 145.1403 & 149.4089 & 3 & 605 \\
\hline 34 & 84.80089 & 72.49063 & 73.9627 & 3.782814 & 230 \\
\hline 35 & 44.8975 & 37.55026 & 37.99172 & 3 & 82 \\
\hline 36 & 52.66131 & 44.28563 & 44.90095 & 3.134719 & 55 \\
\hline 37 & 246.9118 & 218.9643 & 226.593 & 3 & 47 \\
\hline 38 & 52.66131 & 44.28563 & 44.90095 & 3 & 12 \\
\hline 39 & 19.67088 & 15.99184 & 16.00411 & 3 & 8 \\
\hline 40 & 8.758434 & 6.925093 & 6.856592 & 3 & 8 \\
\hline
\end{tabular}




\begin{tabular}{|c|c|c|c|c|c|}
\hline 41 & 16.51591 & 13.34653 & 13.3259 & 3 & 6 \\
\hline 42 & 181.3991 & 159.1714 & 164.0462 & 3.134719 & 45 \\
\hline 43 & 108.1143 & 93.19433 & 95.39297 & 3.134719 & 83 \\
\hline 44 & 116.5742 & 100.7472 & 103.2269 & 3.134719 & 87 \\
\hline 45 & 135.4051 & 117.6252 & 120.7594 & 3.134719 & 106 \\
\hline 46 & 307.2369 & 274.5156 & 284.9027 & 3.134719 & 126 \\
\hline 47 & 84.80089 & 72.49063 & 73.9627 & 3.134719 & 36 \\
\hline 48 & 2413.831 & 2315.072 & 2469.116 & 3.134719 & 1272 \\
\hline 49 & 392.7899 & 353.9322 & 368.5205 & 3 & 156 \\
\hline 50 & 88.92049 & 76.13621 & 77.73109 & 3 & 176 \\
\hline 51 & 33.51367 & 27.74908 & 27.96687 & 3.134719 & 122 \\
\hline 52 & 26.86333 & 22.07421 & 22.18244 & 3 & 41 \\
\hline 53 & 16.51591 & 13.34653 & 13.3259 & 3.270061 & 14 \\
\hline 54 & 13.42202 & 10.76931 & 10.72319 & 3 & 20 \\
\hline 55 & 20.02484 & 16.28958 & 16.30592 & 3 & 18 \\
\hline 56 & 101.3949 & 87.20966 & 89.19133 & 3.270061 & 958 \\
\hline 57 & 60.54499 & 51.1601 & 51.96679 & 3.782814 & 237 \\
\hline 58 & 93.0598 & 79.80509 & 81.52591 & 3 & 130 \\
\hline 59 & 84.80089 & 72.49063 & 73.9627 & 3 & 70 \\
\hline 60 & 21.44701 & 17.48765 & 17.5211 & 3.270061 & 57 \\
\hline 61 & 105.5893 & 90.94392 & 93.06036 & 3 & 50 \\
\hline 62 & 30.16963 & 24.89016 & 25.05064 & 3 & 38 \\
\hline 63 & 33.51367 & 27.74908 & 27.96687 & 3 & 15 \\
\hline
\end{tabular}

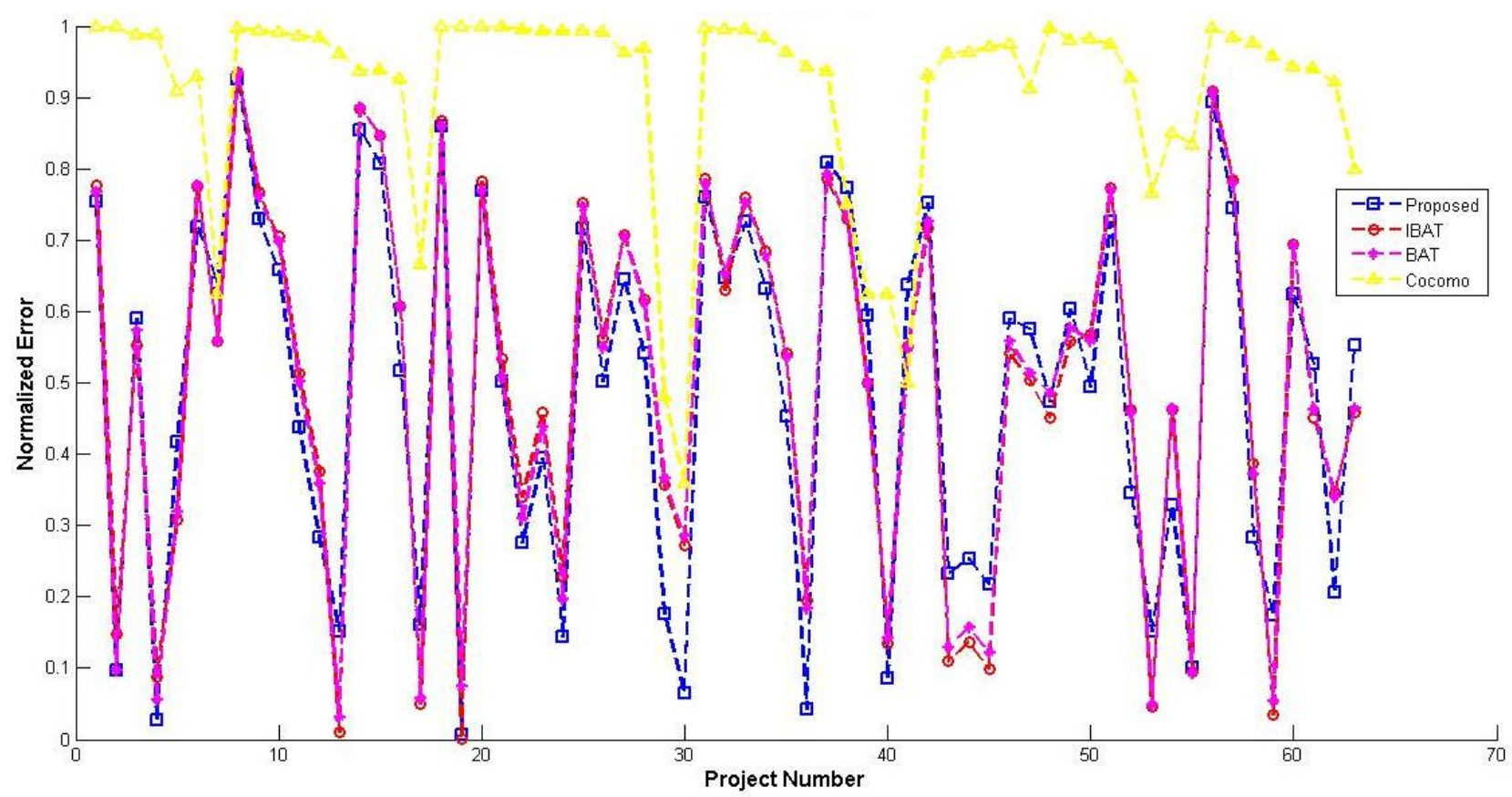

Figure. 1 Analysis of normalized error on Dataset 1 
Table 2. Analysis of effort estimation on Dataset 2

\begin{tabular}{|c|c|c|c|c|c|}
\hline $\begin{array}{l}\text { Project } \\
\text { Number }\end{array}$ & $\begin{array}{l}\text { Hybrid } \\
\text { BATGSA } \\
\text { Optimization }\end{array}$ & $\begin{array}{l}\text { IBAT } \\
\text { Optimization }\end{array}$ & $\begin{array}{l}\text { BAT } \\
\text { Optimization }\end{array}$ & COCOMO & Actual \\
\hline 1 & 287.2043 & 265.0441 & 247.4643 & 3 & 278 \\
\hline 2 & 1167.179 & 1070.171 & 1132.798 & 3 & 1181 \\
\hline 3 & 872.9328 & 801.4547 & 826.5829 & 3 & 1248 \\
\hline 4 & 523.2879 & 481.5761 & 474.4389 & 3 & 480 \\
\hline 5 & 102.5464 & 95.08511 & 80.96048 & 3 & 120 \\
\hline 6 & 63.37579 & 58.8953 & 48.03242 & 3 & 60 \\
\hline 7 & 270.6567 & 249.8417 & 232.0345 & 3 & 300 \\
\hline 8 & 13.85218 & 12.96357 & 9.227148 & 3 & 18 \\
\hline 9 & 29.59828 & 27.60263 & 21.0285 & 3 & 50 \\
\hline 10 & 42.18202 & 39.27361 & 30.88377 & 3 & 60 \\
\hline 11 & 49.45893 & 46.01497 & 36.70414 & 6.520409 & 114 \\
\hline 12 & 16.90391 & 15.80499 & 11.45188 & 6.520409 & 42 \\
\hline 13 & 38.61608 & 35.96821 & 28.06175 & 6.520409 & 60 \\
\hline 14 & 21.65029 & 20.2197 & 14.9788 & 6.520409 & 42 \\
\hline 15 & 387.5022 & 357.109 & 342.4826 & 6.520409 & 450 \\
\hline 16 & 45.79719 & 42.62334 & 33.76551 & 6.520409 & 90 \\
\hline 17 & 138.6684 & 128.3999 & 112.3196 & 6.520409 & 210 \\
\hline 18 & 28.24655 & 26.34772 & 19.98866 & 6.520409 & 48 \\
\hline 19 & 775.5962 & 712.4771 & 727.0806 & 3 & 815 \\
\hline 20 & 185.4654 & 171.5012 & 153.979 & 3 & 239 \\
\hline 21 & 115.5157 & 107.0519 & 92.12647 & 3 & 170 \\
\hline 22 & 37.90906 & 35.31268 & 27.50479 & 3 & 62 \\
\hline 23 & 47.25644 & 43.97509 & 34.93428 & 3 & 70 \\
\hline 24 & 50.56617 & 47.0403 & 37.59643 & 3 & 82 \\
\hline 25 & 127.8651 & 118.441 & 102.8585 & 3 & 192 \\
\hline 26 & 87.81213 & 81.48128 & 68.42088 & 3 & 117.6 \\
\hline 27 & 82.58465 & 76.65239 & 64.01337 & 3 & 117.6 \\
\hline 28 & 20.68617 & 19.32334 & 14.25653 & 3 & 31.2 \\
\hline 29 & 27.23952 & 25.41264 & 19.21672 & 3 & 25.2 \\
\hline 30 & 4.647672 & 4.371511 & 2.821779 & 3 & 8.4 \\
\hline 31 & 8.082867 & 7.58319 & 5.14345 & 3 & 10.8 \\
\hline 32 & 22.29692 & 20.82077 & 15.46477 & 3 & 36 \\
\hline 33 & 270.6567 & 249.8417 & 232.0345 & 3 & 352.8 \\
\hline 34 & 712.3336 & 654.62 & 662.9693 & 6.520409 & 324 \\
\hline 35 & 439.3494 & 404.6549 & 392.4677 & 6.520409 & 360 \\
\hline 36 & 439.3494 & 404.6549 & 392.4677 & 6.520409 & 215 \\
\hline 37 & 439.3494 & 404.6549 & 392.4677 & 6.520409 & 360 \\
\hline 38 & 45.79719 & 42.62334 & 33.76551 & 6.520409 & 48 \\
\hline 39 & 115.0935 & 106.6625 & 91.76123 & 6.520409 & 60 \\
\hline 40 & 110.8853 & 102.7803 & 88.12707 & 6.520409 & 60 \\
\hline 41 & 15.36584 & 14.37324 & 10.32592 & 6.520409 & 24 \\
\hline
\end{tabular}




\begin{tabular}{|l|l|l|l|l|l|}
\hline 42 & 32.67584 & 30.45876 & 23.41075 & 6.520409 & 36 \\
\hline 43 & 64.52773 & 59.96081 & 48.98032 & 6.520409 & 72 \\
\hline 44 & 64.52773 & 59.96081 & 48.98032 & 6.520409 & 48 \\
\hline 45 & 20.04739 & 18.72936 & 13.77956 & 6.520409 & 72 \\
\hline 46 & 1640.226 & 1501.541 & 1638.561 & 6.520409 & 2400 \\
\hline 47 & 2089.383 & 1910.585 & 2130.591 & 6.520409 & 3240 \\
\hline 48 & 1118.321 & 1025.576 & 1081.446 & 6.520409 & 2120 \\
\hline 49 & 192.322 & 177.8117 & 160.1644 & 6.520409 & 370 \\
\hline 50 & 444.5907 & 409.46 & 397.5498 & 10.26826 & 750 \\
\hline 51 & 944.1499 & 866.5265 & 899.9904 & 6.520409 & 420 \\
\hline 52 & 180.9169 & 167.3143 & 149.8864 & 10.26826 & 252 \\
\hline 53 & 68.39128 & 63.53386 & 52.16987 & 6.520409 & 107 \\
\hline 54 & 2450.814 & 2239.436 & 2533.23 & 10.26826 & 2300 \\
\hline 55 & 331.7397 & 305.9395 & 289.3568 & 6.520409 & 400 \\
\hline 56 & 1528.865 & 1400.05 & 1518.225 & 6.520409 & 973 \\
\hline 57 & 1512.239 & 1384.895 & 1500.321 & 6.520409 & 1368 \\
\hline 58 & 326.7409 & 301.3507 & 284.6296 & 10.26826 & 571.4 \\
\hline 59 & 33.02077 & 30.7788 & 23.67897 & 10.26826 & 98.8 \\
\hline 60 & 61.84511 & 57.47932 & 46.77514 & 10.26826 & 155 \\
\hline & & & & & \\
\hline
\end{tabular}

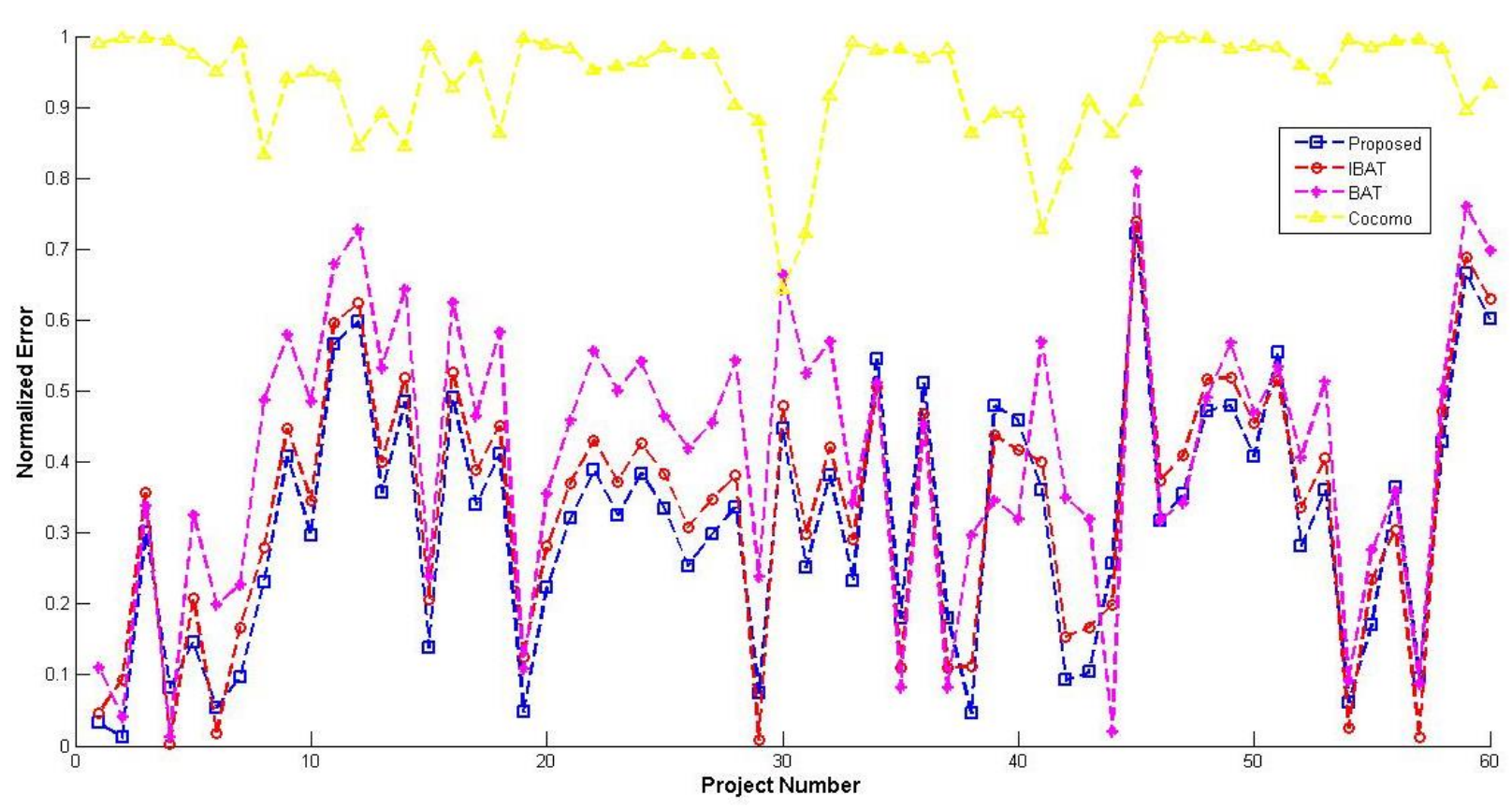

Figure. 2 Analysis of normalized error on Dataset 2

Table 3. Analysis of effort estimation on Dataset 3

\begin{tabular}{|l|l|l|l|l|l|}
\hline $\begin{array}{l}\text { Project } \\
\text { Number }\end{array}$ & $\begin{array}{l}\text { Hybrid } \\
\text { BATGSA } \\
\text { Optimization }\end{array}$ & $\begin{array}{l}\text { IBAT } \\
\text { Optimization }\end{array}$ & $\begin{array}{l}\text { BAT } \\
\text { Optimization }\end{array}$ & COCOMO & Actual \\
\hline 1 & 99.20243 & 93.56455 & 75.74521 & 3 & 117.6 \\
\hline 2 & 93.74146 & 88.38874 & 71.37792 & 3 & 117.6 \\
\hline 3 & 26.13845 & 24.48804 & 18.69947 & 3 & 31.2 \\
\hline 4 & 28.0106 & 26.25112 & 20.10661 & 3 & 36 \\
\hline 5 & 33.69317 & 31.6061 & 24.4048 & 3 & 25.2 \\
\hline
\end{tabular}




\begin{tabular}{|c|c|c|c|c|c|}
\hline 6 & 6.592658 & 6.133727 & 4.409609 & 3 & 8.4 \\
\hline 7 & 10.98438 & 10.24602 & 7.532536 & 3 & 10.8 \\
\hline 8 & 280.2345 & 265.693 & 225.1002 & 3 & 352.8 \\
\hline 9 & 25.39293 & 23.78613 & 18.14048 & 6.520409 & 72 \\
\hline 10 & 74.65834 & 70.3147 & 56.21907 & 6.520409 & 72 \\
\hline 11 & 19.86814 & 18.58797 & 14.02459 & 6.520409 & 24 \\
\hline 12 & 438.1441 & 416.3439 & 359.7068 & 6.520409 & 360 \\
\hline 13 & 39.85181 & 37.41485 & 29.10324 & 6.520409 & 36 \\
\hline 14 & 438.1441 & 416.3439 & 359.7068 & 6.520409 & 215 \\
\hline 15 & 74.65834 & 70.3147 & 56.21907 & 6.520409 & 48 \\
\hline 16 & 438.1441 & 416.3439 & 359.7068 & 6.520409 & 360 \\
\hline 17 & 684.2813 & 651.6947 & 574.1434 & 6.520409 & 324 \\
\hline 18 & 123.0252 & 116.1592 & 94.92732 & 6.520409 & 60 \\
\hline 19 & 54.41322 & 51.16594 & 40.34614 & 6.520409 & 48 \\
\hline 20 & 127.3263 & 120.241 & 98.41099 & 6.520409 & 60 \\
\hline 21 & 73.42792 & 69.15009 & 55.2477 & 3 & 60 \\
\hline 22 & 280.2345 & 265.693 & 225.1002 & 3 & 300 \\
\hline 23 & 114.4643 & 108.0369 & 88.01115 & 3 & 120 \\
\hline 24 & 54.41322 & 51.16594 & 40.34614 & 6.520409 & 90 \\
\hline 25 & 151.2085 & 142.9179 & 117.8548 & 6.520409 & 210 \\
\hline 26 & 34.84069 & 32.68804 & 25.27728 & 6.520409 & 48 \\
\hline 27 & 56.01076 & 52.67581 & 41.5894 & 3 & 70 \\
\hline 28 & 197.7336 & 187.1444 & 156.1494 & 3 & 239 \\
\hline 29 & 59.6202 & 56.08796 & 44.4047 & 3 & 82 \\
\hline 30 & 45.70538 & 42.94007 & 33.60213 & 3 & 62 \\
\hline 31 & 127.7571 & 120.6499 & 98.76027 & 3 & 170 \\
\hline 32 & 140.307 & 132.5641 & 108.9592 & 3 & 192 \\
\hline 33 & 18.05542 & 16.88392 & 12.68562 & 3 & 18 \\
\hline 34 & 36.37604 & 34.13594 & 26.44682 & 3 & 50 \\
\hline 35 & 50.43812 & 47.40997 & 37.26043 & 3 & 60 \\
\hline 36 & 21.69598 & 20.30703 & 15.38079 & 6.520409 & 42 \\
\hline 37 & 46.49121 & 43.6821 & 34.20833 & 6.520409 & 60 \\
\hline 38 & 390.2161 & 370.5845 & 318.552 & 6.520409 & 444 \\
\hline 39 & 27.26034 & 25.54449 & 19.54213 & 6.520409 & 42 \\
\hline 40 & 58.41479 & 54.94832 & 43.46355 & 6.520409 & 114 \\
\hline 41 & 825.4542 & 786.8871 & 698.9662 & 3 & 1248 \\
\hline 42 & 1477.063 & 1412.18 & 1286.772 & 6.520409 & 2400 \\
\hline 43 & 1370.405 & 1309.713 & 1189.493 & 6.520409 & 1368 \\
\hline 44 & 1384.298 & 1323.059 & 1202.144 & 6.520409 & 973 \\
\hline 45 & 338.1075 & 320.866 & 274.0882 & 6.520409 & 400 \\
\hline 46 & 2139.425 & 2049.264 & 1897.824 & 10.26826 & 2400 \\
\hline 47 & 887.3909 & 846.238 & 754.0714 & 6.520409 & 420 \\
\hline 48 & 193.2555 & 182.885 & 152.4424 & 10.26826 & 252 \\
\hline 49 & 78.77285 & 74.20987 & 59.47296 & 6.520409 & 107 \\
\hline
\end{tabular}




\begin{tabular}{|c|c|c|c|c|c|}
\hline 50 & 333.4047 & 316.3806 & 270.0911 & 10.26826 & 571.4 \\
\hline 51 & 40.23975 & 37.78091 & 29.40045 & 10.26826 & 98.8 \\
\hline 52 & 71.79028 & 67.60018 & 53.95606 & 10.26826 & 155 \\
\hline 53 & 442.964 & 420.9472 & 363.8582 & 10.26826 & 750 \\
\hline 54 & 1037.398 & 990.0667 & 888.2906 & 6.520409 & 2120 \\
\hline 55 & 204.468 & 193.5508 & 161.7318 & 6.520409 & 370 \\
\hline 56 & 1079.141 & 1030.109 & 925.815 & 3 & 1181 \\
\hline 57 & 296.004 & 280.7215 & 238.4036 & 3 & 278 \\
\hline 58 & 2.467429 & 2.28434 & 1.573054 & 3 & 8.4 \\
\hline 59 & 5388.899 & 5185.847 & 5000.907 & 10.26826 & 4560 \\
\hline 60 & 1737.146 & 1662.194 & 1525.38 & 6.520409 & 720 \\
\hline 61 & 296.004 & 280.7215 & 238.4036 & 6.520409 & 458 \\
\hline 62 & 1311.233 & 1252.883 & 1135.682 & 6.520409 & 2460 \\
\hline 63 & 390.2161 & 370.5845 & 318.552 & 6.520409 & 162 \\
\hline 64 & 159.9815 & 151.2528 & 125.0365 & 6.520409 & 150 \\
\hline 65 & 619.3632 & 589.5723 & 517.151 & 6.520409 & 636 \\
\hline 66 & 684.2813 & 651.6947 & 574.1434 & 6.520409 & 882 \\
\hline 67 & 1677.211 & 1604.562 & 1470.228 & 6.520409 & 444 \\
\hline 68 & 1147.283 & 1095.493 & 987.223 & 6.520409 & 192 \\
\hline 69 & 654.2464 & 622.9494 & 547.741 & 3 & 576 \\
\hline 70 & 689.2988 & 656.4974 & 578.5597 & 3 & 432 \\
\hline 71 & 133.8024 & 126.3883 & 103.6672 & 3 & 72 \\
\hline 72 & 428.5187 & 407.1519 & 351.4232 & 3 & 300 \\
\hline 73 & 366.4468 & 347.901 & 298.2313 & 3 & 300 \\
\hline 74 & 74.65834 & 70.3147 & 56.21907 & 3 & 240 \\
\hline 75 & 491.4179 & 467.2367 & 405.7102 & 3 & 600 \\
\hline 76 & 744.7064 & 709.5443 & 627.4299 & 3 & 756 \\
\hline 77 & 1748.063 & 1672.694 & 1535.436 & 3 & 1200 \\
\hline 78 & 759.8837 & 724.0786 & 640.8482 & 3 & 97 \\
\hline 79 & 249.8547 & 236.7529 & 199.5761 & 10.26826 & 409 \\
\hline 80 & 438.1441 & 416.3439 & 359.7068 & 10.26826 & 703 \\
\hline 81 & 125.1741 & 118.1984 & 96.66707 & 10.26826 & 1350 \\
\hline 82 & 217.9967 & 206.4237 & 172.9731 & 6.520409 & 480 \\
\hline 83 & 164.3846 & 155.4368 & 128.6483 & 6.520409 & 599 \\
\hline 84 & 91.23059 & 86.0095 & 69.37403 & 6.520409 & 430 \\
\hline 85 & 759.8837 & 724.0786 & 640.8482 & 10.26826 & 4178.2 \\
\hline 86 & 272.8409 & 258.6483 & 218.8754 & 10.26826 & 1772.5 \\
\hline 87 & 296.004 & 280.7215 & 238.4036 & 10.26826 & 1645.9 \\
\hline 88 & 204.468 & 193.5508 & 161.7318 & 10.26826 & 1924.5 \\
\hline 89 & 24.46381 & 22.9115 & 17.44494 & 10.26826 & 648 \\
\hline 90 & 1110.544 & 1060.239 & 954.092 & 10.26826 & 8211 \\
\hline 91 & 59.6202 & 56.08796 & 44.4047 & 10.26826 & 480 \\
\hline 92 & 20.59753 & 19.27386 & 14.56507 & 10.26826 & 12 \\
\hline 93 & 9.271836 & 8.641216 & 6.305753 & 10.26826 & 38 \\
\hline
\end{tabular}




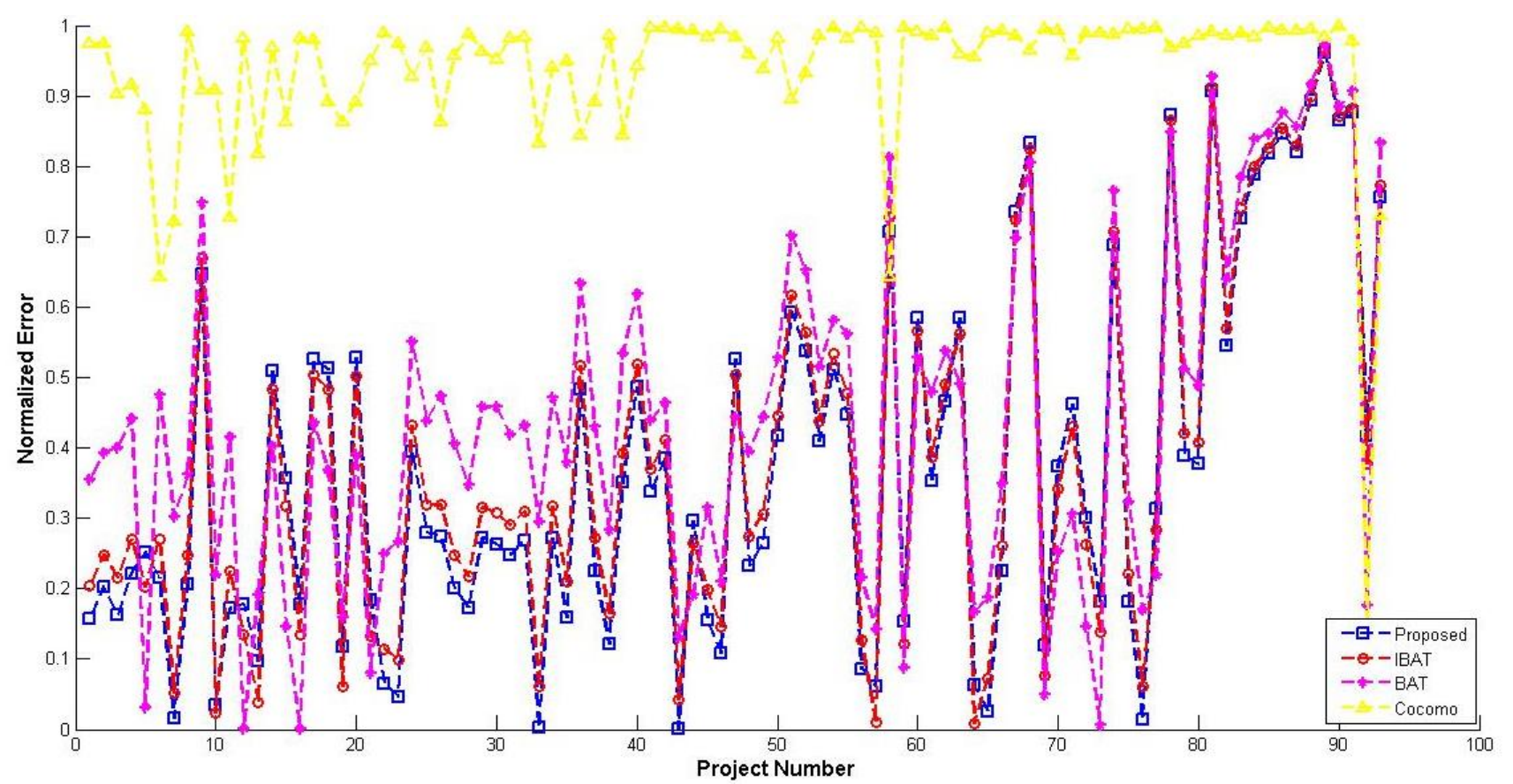

Figure. 3 Analysis of normalized error on Dataset 3

Table 4. Analysis of effort estimation on Dataset 4

\begin{tabular}{|l|l|l|l|l|l|}
\hline $\begin{array}{l}\text { Project } \\
\text { Number }\end{array}$ & $\begin{array}{l}\text { Hybrid } \\
\text { BATGSA } \\
\text { Optimization }\end{array}$ & $\begin{array}{l}\text { IBAT } \\
\text { Optimization }\end{array}$ & $\begin{array}{l}\text { BAT } \\
\text { Optimization }\end{array}$ & COCOMO & Actual \\
\hline 1 & 2617.821 & 4759.931 & 5605.14 & 8564.197 & 287 \\
\hline 2 & 1102.3 & 1961.087 & 2138.508 & 3213.793 & 82.5 \\
\hline 3 & 6374.61 & 11853.65 & 15106.91 & 17526.39 & 1107.31 \\
\hline 4 & 2255.341 & 4085.472 & 4747.639 & 5266.707 & 86.9 \\
\hline 5 & 4273.649 & 7867.239 & 9676.503 & 9519.336 & 336.3 \\
\hline 6 & 981.854 & 1741.718 & 1879.869 & 2610.139 & 84 \\
\hline 7 & 203.2856 & 346.5822 & 325.2334 & 520.7564 & 23.2 \\
\hline 8 & 2583.915 & 4696.738 & 5524.325 & 6818.744 & 130.3 \\
\hline 9 & 4188.328 & 7706.258 & 9461.534 & 11576.37 & 116 \\
\hline 10 & 570.9059 & 998.9934 & 1027.525 & 1389.818 & 72 \\
\hline 11 & 4332.574 & 7978.464 & 9825.252 & 11723.8 & 258.7 \\
\hline 12 & 1820.726 & 3280.442 & 3740.319 & 5270.448 & 230.7 \\
\hline 13 & 1768.673 & 3184.33 & 3621.389 & 4542.2 & 157 \\
\hline 14 & 3664.985 & 6720.702 & 8154.226 & 9598.281 & 246.9 \\
\hline 15 & 2521.852 & 4581.12 & 5376.708 & 7214.489 & 69.9 \\
\hline
\end{tabular}

\section{Conclusion}

This paper implements the hybrid BATGSA algorithm to optimize the performance of the COCOMO model to estimate the software cost. The algorithm has been analyzed on 4 datasets including two datasets of NASA projects downloaded from promise repository. The comparison of effort valueand normalized error on each dataset has been done with the Improved BAT, BAT and COCOMO model. The normalized error has been reduced by $1 \%$ on dataset $1,3.5 \%$ on dataset $2,0.33 \%$ on dataset 3 and $46 \%$ on dataset 4 by BATGSA algorithm as compared to the improved BAT algorithm. The reduction in the normalized error and the convergence of efforts towards the actual effort of 


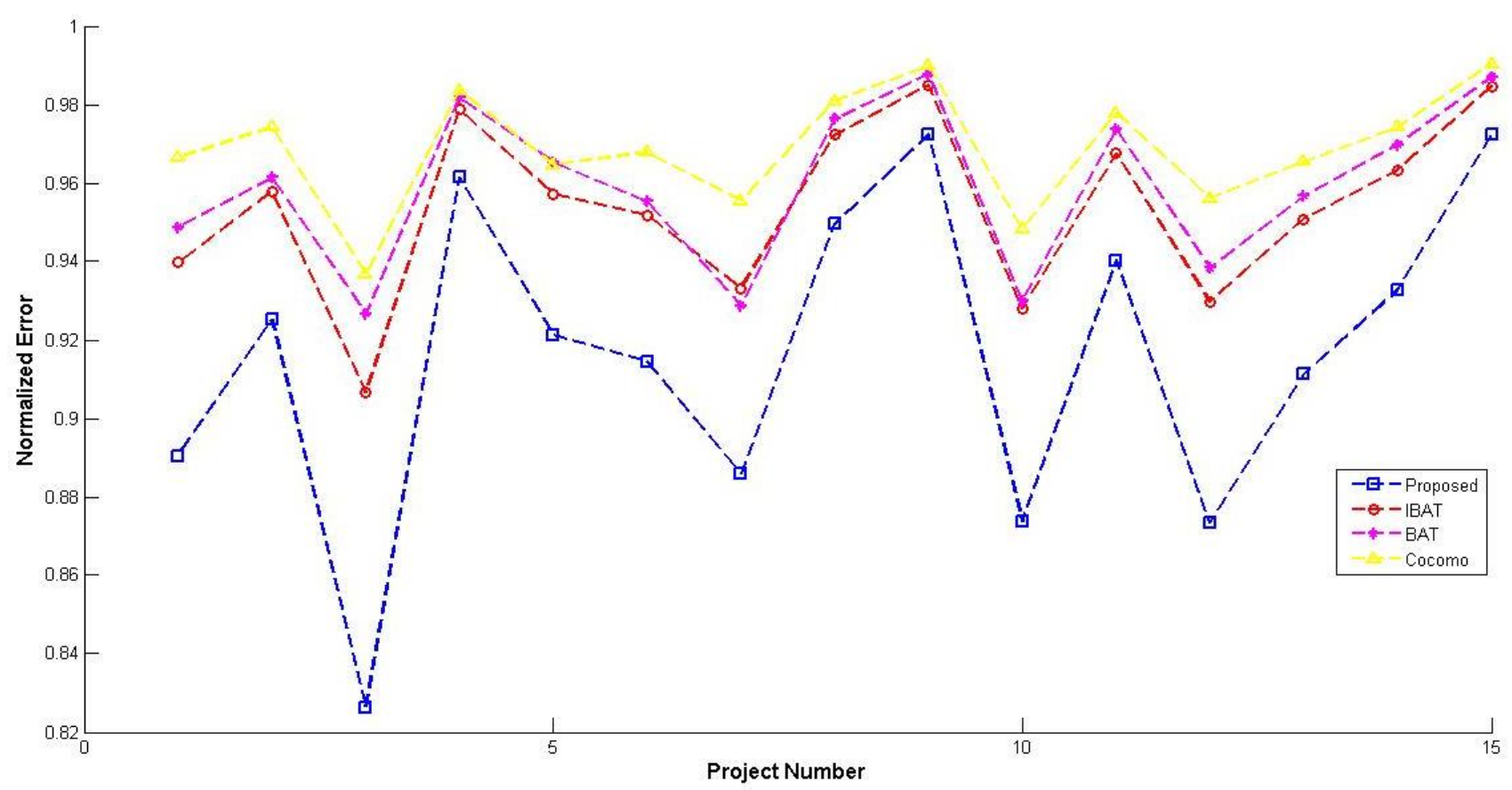

Figure. 4 Analysis of normalized error on Dataset 4

the hybrid BATGSA algorithm as compared to other state of art algorithms proves the significance of the algorithm. In future this algorithm can be applied to optimize different software metrics. Moreover, algorithm can be modified to determine the software quality during the development of software.

\section{References}

[1] B. W. Boehm, "Software cost estimation meets software diversity", In: Proc. of the 2017 IEEE/ACM 39th International Conference on Software Engineering Companion, ICSE-C 2017, pp. 495-496, 2017.

[2] S. Aljahdali and A. F. Sheta, "Software effort estimation by tuning COOCMO model parameters using differential evolution", In: Proc. of the ACS/IEEE Int. Conf. Comput. Syst. Appl. - AICCSA 2010, pp. 1-6, 2010.

[3] I. M. Keshta, "Software Cost Estimation Approaches: A Survey", J. Softw. Eng. Appl., Vol. 10, No. 10, pp. 824-842, 2017.

[4] R. Wang, P. Peng, L. Xu, X. Huang, and X. Qiao, "A Novel Algorithm for Software Development Cost Estimation Based on Fuzzy Rough Set", J. Eng. Sci. Technol. Rev., Vol. 9, No. 4, pp. 217223, 2016.

[5] A. H. Gandomi, X. S. Yang, S. Talatahari, and A. H. Alavi, "Metaheuristic Algorithms in Modeling and Optimization", Metaheuristic Applications in Structures and Infrastructures, pp. 1-24, 2013.
[6] A. F. Sheta, "Estimation of the COCOMO model parameters using genetic algorithms for NASA software projects", J. Comput. Sci., Vol. 2, No. 2, pp. 118-123, 2006.

[7] N. Gupta, "Optimizing Intermediate COCOMO Model Using BAT Algorithm", In: Proc. of the 2nd Int. Conf. Comput. Sustain. Glob. Dev., p. 1649, 2015.

[8] J. D. Altringham, Bats: biology and behaviour. Oxford Univesity Press, 1996.

[9] X. S. Yang, "A new metaheuristic Bat-inspired Algorithm", Studies in Computational Intelligence, Vol. 284, pp. 65-74, 2010.

[10] K. Kiełkowicz and D. Grela, "Modified Bat Algorithm for Nonlinear Optimization", IJCSNS Int. J. Comput. Sci. Netw. Secur., Vol. 16, No. 10, pp. 46-50, 2016.

[11] S. Girotra and K. Sharma, "Tuning of Software Cost Drivers using BAT Algorithm", In: Proc. of the 10th INDIACOM - 20163 rd international conference on computing for sustainable global development, pp. 1051-1056, 2016.

[12] E. Rashedi, H. Nezamabadi-pour, and S. Saryazdi, "GSA: A Gravitational Search Algorithm", Inf. Sci. (Ny)., Vol. 179, No. 13, pp. 2232-2248, 2009. 